\title{
Knowledge of General Physicians About Urine Analysis: A Cross Sectional Study in Tehran, Iran
}

\author{
Faezeh Javadi Larijani ${ }^{1}$, Elham Khatooni ${ }^{2}$, Siamak Amiri ${ }^{3,{ }^{*}}$, Seyed Hassan Saadat ${ }^{4}$, Alipasha \\ Meysamie $^{5}$, Ali Asghar Akhlaghi ${ }^{6, * *}$ and Ali Arasteh ${ }^{7}$ \\ ${ }^{1}$ Health Policy Research Center, Institute of Health, Shiraz University of Medical Sciences, Shiraz, Iran \\ ${ }^{2}$ Department of Epidemiology and Biostatistics, School of Public Health, Tehran University of Medical Sciences, Tehran, Iran \\ ${ }^{3}$ Tehran University of Medical Sciences (TUMS), Tehran, Iran \\ ${ }^{4}$ Behavioral Sciences Research Center, Lifestyle Institute, Baqiyatallah University of Medical Sciences, Tehran, Iran \\ ${ }^{5}$ Community and Preventive Medicine Department, Medical Faculty, Tehran University of Medical Sciences, Tehran, Iran \\ ${ }^{6}$ Department of Epidemiology and Reproductive Health, Reproductive Epidemiology Research Center, Royan Institute for Reproductive Biomedicine, ACECR, Tehran, Iran \\ ${ }^{7}$ Faculty of Islamic Studies Department, Imam Khomeini Internation University (IKIU), Qazvin, Iran \\ *Corresponding author: Tehran University of Medical Sciences (TUMS), Tehran, Iran. Email: epedneph18@gmail.com \\ ${ }^{* *}$ Corresponding author: Department of Epidemiology and Reproductive Health, Reproductive Epidemiology Research Center, Royan Institute for Reproductive Biomedicine, \\ ACECR, P.O. Box: 16635-148, Tehran, Iran. Email: akhlaghi90@gmail.com
}

Received 2018 February 03; Revised 2018 May 20; Accepted 2018 May 22.

\begin{abstract}
Background: Urine is the most commonly used liquid for diagnosis and prognosis of diseases and is still the only body fluid that is applied to many diagnostic purposes. There is little information of physicians' knowledge about correct urine sampling, interpretation in Iranian.

Objectives: Evaluation the knowledge of the physicians in urine analysis and identifying related factors to provide useful measures and recommendations to raising physicians' knowledge.

Methods: This cross-sectional study was conducted on 272 physicians who were attended to the congress of retraining in Tehran (capital of Iran) in 2015. The first questionnaire was demographic and the second one was researcher-made consisting of 30 multiple choices questions in 3 different sections. Statistical analysis was performed using STATA 13 software.

Results: The Cronbach's alpha of the researcher-made questionnaire was $76 \%$ and response rate was $45.3 \%$. Most of the physicians were in the moderate level in terms of knowledge about sampling for urine analysis (62.5\%), knowledge about interpretation of the urine analysis results (54.8\%) and knowledge to link urinalysis result with the patient's clinical symptoms (73.5\%). Knowledge was reduced in male sex, increasing in age and time elapsed since graduation and increased with more time studying medicine, and attending in retraining congresses.

Conclusions: It is recommended that physician, especially, who have long passed their graduation, spend more time studying medicine and if possible, take part in the congresses and retraining program to keep their information update and apply best diagnostic treatments to their patients.
\end{abstract}

Keywords: Urine Test, Validity, Urine Questionnaire, Factor Structure

\section{Background}

Urine is a sterile fluid, which is a result of the accumulation of body excreted substances that are secreted by the kidneys and accumulated in the bladder and repelled through the urethra (1). Urine is the most commonly used liquid for diagnosis and prognosis of diseases and is still the only body fluid that is applied to many diagnostic purposes (2). Urinalysis is one of the easiest and, at the same time, most practicing doctor-assisted diagnostic test and also early detection of some diseases by urinalysis is a key to influencing and reducing the cost of treatment (3). A complete urinalysis test includes physical, chemical, and microscopic examination of the urine (3). Urinalysis usually is done in the urinary tract infections (kidneys, ureter and bladder), determine the presence of sugar in diabetic patients, kidney stones, high blood pressure, and certain kidney and liver diseases (4).

There is little information of Iranian physicians' knowledge about correct urine sampling, interpretation of urine test results and the knowledge to link urinalysis result with the patient's clinical symptoms.

By evaluating the level of knowledge of physicians in urine sampling and interpreting and also, identifying 
their weaknesses in the correct interpretation of urinalysis, in could be possible to provide useful measures and recommendations to raising physicians knowledge of urine sampling and interpretation of urine test results.

\section{Methods}

\subsection{Subject}

This cross-sectional study was conducted on 272 general practitioners who were attended to the congress of retraining in Tehran (capital of Iran) in 2015. This research was approved by Ethics Committee of Tehran University of Medical Sciences, No: IR.TUMS.REC.1395.2859.

\subsection{Survey Instrument}

A data collection tool was consists of two parts: The first part include questions related to the demographic characteristics of physicians and the second part is a researcher-made questionnaire consisting of 30 multiple choices questions with 4 options in 3 different sections, each of these questions have only one correct answer, and all of the questions have similar weight to calculate total score. Also, the questionnaire was developed after sufficient literature review. Definition of 3 sections is given below:

Section 1: questions from 1 to question 10 are related to physicians' knowledge of correct method for urine sampling.

Section 2: questions from 11 to question 20 are related to physicians' knowledge of correct interpretation of urine test results.

Section 3: questions from 21 to question 30 are related to physicians knowledge to link urinalysis result with the patient's clinical symptoms.

Also, the score of each physician in each section was placed to one of the 3 following categories:

Correct answer to at least 7 questions in each section is considered as "good" knowledge.

Correct answer to at least 4 questions and up to 6 questions in each section is considered as "moderate" knowledge.

Correct answer up to 3 questions in each section is considered as "bad" knowledge.

\subsection{Statistical Methods}

\subsubsection{Validation of the Questionnaire}

Reliability: The reliability of the questionnaire has been investigated by use the Cronbach's alpha.

Content validity: The appropriateness of the items and the triple domains were evaluated, according to the opinion of three nephrology specialists.
Structure validity: Spearman correlation matrix was first calculated for triple scores, and then the KMO (KaiserMeyer-Olkin) index and the Bartlett test were used to determine the suitable of the data for factor analysis.

The results were expressed for quantitative variables as mean (standard deviation: SD), and for the categorical variables as frequency, percentage. Statistical analysis of data was performed by t-test and Chi-square test using STATA 13 software. Also, P value less than 0.05 was considered as statistically significant.

\section{Results}

A total of 600 questionnaires were distributed among general physicians who were attended at the congress with retaining score in Tehran, Iran in 2015. Out of total 600 physicions, 272 completed questionnaires were returned to the researcher resulted in $45.3 \%$ response rate. Out of total 272 physicians entered to the study, 128 (47.1\%) of them were male. The mean age mean (SD) of physicians physician was 32.65 (8.65) years. Also, 87 (33.7\%) physicians were graduated from Tehran University of Medical Sciences. According to the classification of universities in Iran, 141 of the physicians (54.7\%) were graduated from level 1 (best level) universities, 84 (32.5\%) from level 2 and 33 (12.8\%) from level 3 universities.

161 physicians (64.4\%) selected Tehran as their place of employment. In term of service place type, the highest number was for the 62 physician, who were either family medicine or medical service plan. The mean (SD) of the year's number of service was 8.65 (7.5) years, with minimum and maximum of 6 months and 30 years, respectively. The mean (SD) of working hours of physicians per week was 38.81 (17.74) hours, ranging from 4 to 84 hours. Some physicians had not already attended another retraining congress, while others attended a maximum of 6 other programs, the mean (SD) number of retraining programs was 2.57 (1.48).

The mean (SD) of physicians response to the question "Out of 10 patients who are referred to you, for how many of them urine analysis should be requested?" was $1.72(2.62)$.

\subsection{Validity and Reliability of the Questionnaire}

Descriptive statistics, correlation matrix, Cronbach's alpha and measures for structural validity of 3 domains of researcher-made questionnaire for evaluation of physicians' knowledge about urinalysis were reported in the Table 1.

The linear correlation of the scores in the triple domains was about 50\%. The Cronbach's alpha of the general 
Table 1. Descriptive Statistics, Correlation Matrix, Cronbach's Alpha and Measures for Structural Validity of 3 Domains of Researcher-Made Questionnaire for Evaluation of Physicians' Knowledge About Urinalysis

\begin{tabular}{|c|c|c|c|c|c|c|c|c|c|c|}
\hline & \multicolumn{3}{|c|}{ Correlation Matrix } & \multirow[t]{2}{*}{ Alpha } & \multirow[t]{2}{*}{ КМо } & \multirow{2}{*}{$\begin{array}{l}\text { 1st Factor } \\
\text { Loading }\end{array}$} & \multirow[t]{2}{*}{ No. } & \multirow[t]{2}{*}{ Mean \pm SD } & \multirow[t]{2}{*}{ Median } & \multirow[t]{2}{*}{ IQR } \\
\hline & $\begin{array}{c}\text { Sampling } \\
\text { Knowledge }\end{array}$ & $\begin{array}{c}\text { Interpretation } \\
\text { Knowledge }\end{array}$ & $\begin{array}{c}\text { Compliance } \\
\text { with Clinical } \\
\text { Symptoms }\end{array}$ & & & & & & & \\
\hline $\begin{array}{l}\text { Sampling } \\
\text { knowledge }\end{array}$ & 1 & - & - & 0.67 & 0.70 & 0.83 & 270 & $4.25 \pm 1.74$ & 4 & $3-5$ \\
\hline $\begin{array}{l}\text { Interpretation } \\
\text { knowledge }\end{array}$ & $0.54^{* *}$ & 1 & - & 0.65 & 0.68 & 0.84 & 261 & $5.0 \pm 2.25$ & 5 & $3-7$ \\
\hline $\begin{array}{l}\text { compliance } \\
\text { with clinical } \\
\text { symptoms }\end{array}$ & $0.50^{* *}$ & $0.54^{* *}$ & 1 & 0.70 & 0.72 & 0.81 & 264 & $4.9 \pm 1.59$ & 5 & $4-6$ \\
\hline Total score & - & - & - & 0.76 & 0.70 & - & 261 & $14.58 \pm 4.63$ & 15 & $11-18$ \\
\hline
\end{tabular}

Abbreviations: IQR, inter quartile range; SD, standard deviation.

Table 2. Number (\%) of Physicians in Each Response Category, Separately, for 3 Domains of Questionnaire ${ }^{\mathrm{a}}$

\begin{tabular}{lccc}
\hline Domain & Good & Moderate & Bad \\
\hline Sampling knowledge & $5(1.8)$ & $170(62.5)$ & $97(35.7)$ \\
Interpretation knowledge & $39(14.3)$ & $149(54.8)$ & $84(30.9)$ \\
$\begin{array}{l}\text { Compliance with clinical } \\
\text { symptoms }\end{array}$ & $19(7.0)$ & $200(73.5)$ & $53(19.5)$ \\
\hline
\end{tabular}

${ }^{\mathrm{a}}$ Values are expressed as No. (\%).

30 items questionnaire was 76\%, which indicates the appropriate reliability of the researcher-made questionnaire. Also, the KMO index was 0.70 and Bartlet test was significant $(\mathrm{P}<0.001)$, which indicates that the suitability of the questionnaire structure and presence the latent factor in the triple domains of the questionnaire. Factor analysis also showed that only the first factor has eigen value greater than one, so the one-factor model is suitable (Table 1).

Number (\%) of physicians in each response category, separately, for 3 domains of questionnaire are given in the Table 2. Generally, most of the physicians score in the triple domains of questionnaire were placed in "bad" or "moderate" knowledge categories (Table 2).

Descriptive statistics for total score of questionnaire based of physicians' characteristics with statistical test of association between physicians' characteristics and total score of questionnaire are given in the Table 3.

The mean (SD) of total score of physician knowledge about urinalysis was 14.13 (4.87).

The association between the physicians' characteristics was assessed by the total score of the physicians. Based on this, the mean score of female physicians was significantly higher than males $(\mathrm{P}=0.033)$. On the other hand, with an increase in the time elapsed since graduation, the mean of total score was significantly reduced. So that the lowest mean score was observed by the physicians who had passed more than 10 years of their graduation, while the highest mean score was obtained by the physicians who have been less than 5 years since their graduation ( $P$ $<0.001$ ). The differences in terms of service type were also statistically significant. So the lowest mean score was obtained by physicians who worked in a private hospital while those who in the urban health centers had the highest mean score, after which the physician who worked at the government hospitals had the highest mean (Table 3 ).

In terms of quantitative variables, an inverse significant correlation was found between the total score and the age of the physicians, so that the score decreased with increasing age $(\mathrm{P}<0.001)$. There was also an inverse association between the total score and the year's number of service $(\mathrm{P}<0.001)$. On the other hand, there was a direct correlation between the number of hours worked per week ( $\mathrm{P}$ $=0.008)$, the number of retraining sessions $(P=0.026)$ and the number of study hours in the field of medicine per day $(\mathrm{P}=0.005)$ with the overall score (Table 3$)$.

Descriptive statistics for triple domains of questionnaire based on physicians' characteristics with statistical test and Pvalue are given in the Table 4 . The association patterns of physicians' characteristics with each of the triple domains of physicians' knowledge about urine analysis were similar to the association reported for the total score in the Table 3.

\section{Discussion}

In this study, 272 physicians completed a questionnaire of urine analysis knowledge and response rate was $45 \%$. Most of the physicians were in the moderate level in terms of knowledge about sampling for urine analysis (62.5\%), knowledge about how to interpret the results of urine analysis (54.8\%) and the level of physicians ' knowledge to link urinalysis result with the patient's clinical symptoms 
Table 3. Descriptive Statistics for Total Score of Questionnaire Based on Physicians Characteristics with Statistical Test

\begin{tabular}{|c|c|c|}
\hline Variables & Overall Score, Mean \pm SD & PValue \\
\hline \multicolumn{3}{|c|}{ Qualitative } \\
\hline Sex & & 0.033 \\
\hline Male & $13.49 \pm 4.74$ & \\
\hline Female & $14.77 \pm 4.95$ & \\
\hline University level & & 0.722 \\
\hline 1 & $14.49 \pm 5.04$ & \\
\hline 2 & $14.15 \pm 4.90$ & \\
\hline 3 & $13.79 \pm 3.94$ & \\
\hline Years after graduation, $y$ & & $<0.001$ \\
\hline$<5$ & $14.98 \pm 5.05$ & \\
\hline $5-10$ & $13.04 \pm 3.78$ & \\
\hline$>10$ & $12.43 \pm 3.96$ & \\
\hline Type of service place & & $<0.001$ \\
\hline Personal clinic & $13.05 \pm 3.83$ & \\
\hline Private hospital & $10.12 \pm 3.14$ & \\
\hline General hospital & $15.67 \pm 5.25$ & \\
\hline Rural health house & $14.25 \pm 4.35$ & \\
\hline Urban health center & $16.17 \pm 4.49$ & \\
\hline Family physician & $14.18 \pm 4.91$ & \\
\hline \multirow[t]{3}{*}{ Health } & $14.35 \pm 4.82$ & \\
\hline & \multicolumn{2}{|c|}{ Quantitative } \\
\hline & Spearman Rank Correlation & PValue \\
\hline Age & -0.245 & $<0.001$ \\
\hline Years of service & -0.277 & $<0.001$ \\
\hline Work (hours/week) & 0.201 & 0.008 \\
\hline Retraining session & 0.207 & 0.026 \\
\hline $\begin{array}{l}\text { Literature review } \\
\text { (hours/week) }\end{array}$ & 0.192 & 0.005 \\
\hline $\begin{array}{l}\text { Patient related urine } \\
\text { analysis }\end{array}$ & 0.053 & 0.429 \\
\hline
\end{tabular}

(73.5\%). Also, the lowest percentage of physicians were in the category of "good knowledge" in all of the triple domains (Table 2).

Studies on the large sample of physicians in other countries also revealed lack of update information about urine analysis and poor compliance of physicians with global recommendations for urine sampling and interpretation (5-7).

There is a notable variation between nephrologists and gold standard urine analysis in term of perform and its interpretation, which should be more emphasized on the principles and importance of laboratory tests, especially urinary analysis (8). Contrary to international guidelines that do not recommend routine use of dipstick for screening, occupation physician in Belgium are doing their job to meet their patients' demands. Additionally, there is insufficient knowledge about the positivity of tests and referral criteria for patients to the specialist (5).

The response rate of physician in this study was about $45 \%$. In the study of Drekonja et al. (6) the response rate was $36 \%$ and about $48 \%$ of the residents answered correctly, but in the present study, good knowledge in the three domains of sampling, interpretation, and the ability of physicians to link urinalysis result with the patient's clinical symptoms were $2 \%, 14 \%$ and $7 \%$, respectively. So, good knowledge in the triple domains of the questionnaire was low and most of the physicians had a moderate level of knowledge in the triple domains (Table 2).

In the study of Derkonja et al. there was an association between practice experience and demographic factors with the general knowledge of residents. For example, residents at year 3 , were significantly more knowledgeable than others (6). Also, in the present study, significant association was observed for total and triple scores of physicians' knowledge with physicians' characteristics (Tables 3 and 4 ).

In the study of Jones et al. (9), the knowledge and practice of 394 nurses from 5 hospitals about the causes and methods of urine sampling were assessed by using selfmade questionnaires with 40 questions and 58\% nurse's compliance has been achieved with gold standard guideline.

Published literature indicates that the unjustified ordering or improper collection of urine for urinalysis or culture from either catheterized patients or those without indwelling devices, or misinterpretation of positive results, often leads to adverse health care events, including increased financial burdens, over reporting of mandated catheter-associated urinary tract infection events, overtreatment of patients with antimicrobial agents, selection of multidrug-resistant organisms, and Clostridium difficult infection (10).

The correct interpretation of the urine analysis results and the ability of physicians to link urinalysis result with the patient's clinical symptoms is important in terms of the health economy. In fact, both the correct referee of people for urinalysis and compliance with the guidelines in sampling and final interpretation of urine analysis result are important because misinterpretation and unjustified ordering or improper urine sample collection could be resulted in economic costs, complication in person's health and even an infection or overtreatment (10).

Meta-analysis study by Flokas et al. (7) showed that the mishandling of asymptomatic bacteriuria (ASB) is fre- 
quent. There were an association between female sex and the over interpretation of special laboratory result with overtreatment. Antimicrobial supervision plans can be effective and should focus on various interpreting of urine infection from ASB. Also, Flokas et al. showed that the implementation of educational programs can reduce the overtreatment median absolute risk by $33 \%$ (7).

The physicians of this study may not be representative of physicians' population in Tehran, because of sampling them from congress and the risk of selection bias which could be considered as a limitation of this study.

Considering the importance of urine analysis and the consequences of inappropriate knowledge for conducting this test, it is necessary to complete the training needed to raise knowledge, especially, in the subgroups of physicians who are significantly have less knowledge than other.

\subsection{Conclusion}

It is recommended that physician, especially, who have long passed their graduation, spend more time studying medicine and if possible, take part in the congresses and retraining program to keep their information update and apply best diagnostic treatments to their patients.

\section{Acknowledgments}

The researchers, thus, acknowledge their great gratitude to the physicians who participated in this study. We sincerely thank respectful reviewer (s) for their valuable comments.

\section{Footnotes}

Authors' Contribution: Study concept and design, Faezeh Javadi Larijani, Siamak Amiri; acquisition of data, Seyed Hassan Saadat, Ali Arasteh; analysis and interpretation of data, Seyed Hassan Saadat, Ali Arasteh; drafting of the manuscript, Elham Khatooni, Ali Akhlaghi; statistical analysis, Alipasha Meysamie, Ali Asghar Akhlaghi; administrative, technical, and material support, Faezeh Javadi Larijani, Siamak Amiri. All authors evaluated and edited the manuscript.

Conflict of Interests: The authors declare that they have no conflicts of interest.

\section{References}

1. Gerber GS, Brendler CB. Evaluation of the urologic patient: history, physical examination, and urinalysis. Campbell-Walsh urology. 9 th ed. Philadelphia, Pa: Saunders Elsevier; 2007.

2. White WI. A new look at the role of urinalysis in the history of diagnostic medicine. Clin Chem.1991;37(1):119-25. [PubMed: 1988194].

3. Simerville JA, Maxted WC, Pahira JJ. Urinalysis: a comprehensive review. Am Fam Physician. 2005;71(6):1153-62. [PubMed: 15791892].

4. Reine NJ, Langston CE. Urinalysis interpretation: how to squeeze out the maximum information from a small sample. Clin Tech Small Anim Pract.2005;20(1):2-10. doi:10.1053/j.ctsap.2004.12.002. [PubMed: 15822525].

5. Braeckman L, Haak E, Peremans L. Routine dipstick urinalysis in daily practice of Belgian occupational physicians. Arch Public Health. 2012;70(1):15. doi: 10.1186/0778-7367-70-15. [PubMed: 22958323]. [PubMed Central: PMC3436716].

6. Drekonja DM, Abbo LM, Kuskowski MA, Gnadt C, Shukla B, Johnson JR. A survey of resident physicians' knowledge regarding urine testing and subsequent antimicrobial treatment. Am J Infect Control. 2013;41(10):892-6. doi: 10.1016/j.ajic.2013.01.020. [PubMed: 23541740].

7. Flokas ME, Andreatos N, Alevizakos M, Kalbasi A, Onur P, Mylonakis E. Inappropriate management of asymptomatic patients with positive urine cultures: A systematic review and meta-analysis. Open Forum Infect Dis. 2017;4(4):ofx207. doi: 10.1093/ofid/ofx207. [PubMed: 29226170]. [PubMed Central: PMC5714225].

8. Cushner HM, Copley JB. Back to basics: the urinalysis: a selected national survey and review. Am J Med Sci. 1989;297(3):193-6. doi: 10.1097/00000441-198903000-00015. [PubMed: 2646921].

9. Jones K, Sibai J, Battjes R, Fakih MG. How and when nurses collect urine cultures on catheterized patients: A survey of 5 hospitals. Am JInfect Control.2016;44(2):173-6. doi:10.1016/j.ajic.2015.09.003. [PubMed: 26492819].

10. Garcia R, Spitzer ED. Promoting appropriate urine culture management to improve health care outcomes and the accuracy of catheter-associated urinary tract infections. Am J Infect Control. 2017;45(10):1143-53. doi: 10.1016/j.ajic.2017.03.006. [PubMed: 28476493]. 


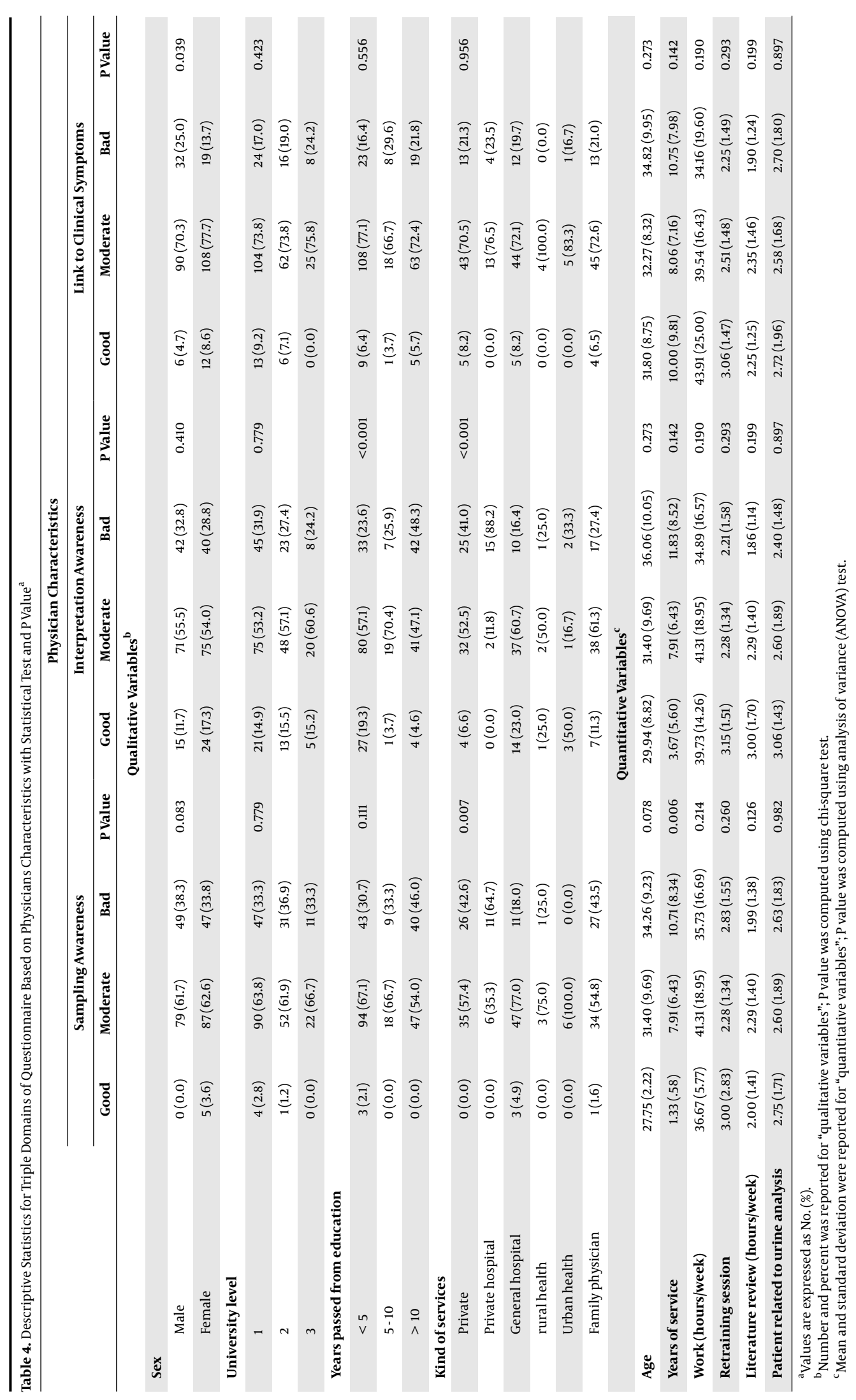

